\title{
De Novo Mutation in SCN4A Gene Detected in Polish Patient with Paramyotonia Congenita Phenotype
}

\author{
Barbara Zapala $^{1 *}$, Agnieszka Spychałowicz, Urszula Ciałowicz ${ }^{1}$, Zbigniew Żuber ${ }^{2}$, Bogdan Solnica ${ }^{1}$ \\ ${ }^{1}$ Department of Clinical Biochemistry, Jagiellonian University, Medical College, Cracow, Poland \\ ${ }^{2}$ Department of Rheumatology, St Louis Voivodeship Specialist Children's Hospital, Cracow, Poland
}

"Corresponding author: Barbara Zapała, Department of Clinical Biochemistry, Jagiellonian University, Medical College, Kopernika str.15A, Kraków, 31-501, Poland. Tel: +48124248787, Fax:+48124248797: Email: barbara.zapala@uj.edu.pl

Citation: Zapała B, Spychałowicz A, Ciałowicz U, Żuber Z, Solnica B (2017) De Novo Mutation in SCN4A Gene Detected in Polish Patient with Paramyotonia Congenita Phenotype. Int J Genom Data Min 01: 109. DOI: 10.29011/2577-0616.000109

Received Date: 01 July, 2017; Accepted Date: 04 July, 2017; Published Date: 11 July, 2017

\begin{abstract}
A 16-year-old Polish male child was referred to confirm the diagnosis of myotonia congenita. For several years he had experienced cold-induced myotonia and muscle stiffness. DNA analysis of the $S C N 4 A$ gene showed a C to T transition at nucleotide position 3938 in exon 22 of $S C N 4 A$ (Thrl313Met) in patient but not in his parents. Based on his symptoms and results of DNA analysis, paramyotonia congenita was diagnosed, which prevalence is very low in Poland.
\end{abstract}

Keywords: Missense Mutation; Paramyotonia Congenita SCN4A gene; Sodium Channel

\section{Introduction}

Paramyotonia congenita (PMC; OMIM \#168300), first described by Eulenburg [1] represents a skeletal muscle sodium channelopathy due to episodic membrane hyperexcitability of the skeletal muscle cells. This disorder has an autosomal dominant mode of inheritance with a variable penetration. Clinical symptoms are episodic and from one patient to another may vary in severity, duration and frequency. Symptomatically, PMC is characterised by paradoxical and cold-induced myotonia, defined as increased stiffness during repeated activities and cold-induced muscle stiffness. PMC usually affects the neck, face and distal upper extremity muscles. This muscle stiffness is induced by exercise or exposure to cold. However, the phenotype of PMC is very heterogenous; in some patients paramyotonia occurs during exercise even under warm temperatures, some others have cold-induced paralytic attacks without stiffness whilst in others stiffness can be induced by cold temperature without weakness [2-6]. PMC is caused by a mutation in the $S C N 4 A$ gene. $S C N 4 A$ gene is located on chromosome $17 \mathrm{q} 23-25$ and comprises 24 exons with a $5.5-\mathrm{kb}$ open reading frame. This gene encodes a skeletal muscle voltage-gated sodium channel, Nav 1.4. The protein encoded by the SCN4A gene comprises 1836 amino acids and mediates the voltage-dependent permeability of the excitable membrane for sodium ion. The ex- pression of Nav 1.4 has been detected in all skeletal muscle cells. During the past 30 years more than 50 different types of $S C N 4 A$ mutation from several populations and with several clinically different forms of myotonia have been identified, most of which are single-base substitutions producing missense mutations. Studies have shown that certain $S C N 4 A$ mutations are associated with a specific phenotype [7-10].

\section{Case Study}

The patient was 16-year old male child that was referred to us from another clinic to confirm the diagnosis of paramyotonia congenita. He was the only child and at age 12 years he began to experience muscle stiffness that affected his face and upper and lower extremities. Both muscle stiffness and weakness were provoked and worsened by muscle cooling (such as being outdoors in winter temperatures of $5^{\circ} \mathrm{C}-10^{\circ} \mathrm{C}$ ) and muscle activity. Routine laboratory tests were performed including complete blood count, liver and renal function tests, electrolytes and serum Creatine Kinase (CK) level. The laboratory findings were all nearly normal, serum sodium potassium and chloride levels were normal, although CK level was elevated at $502 \mathrm{U} / \mathrm{L}$, two months later at $1008 \mathrm{U} / \mathrm{L}$, and after one year at $914 \mathrm{U} / \mathrm{L}$ (normal range: $0.00-171.00 \mathrm{IU} / \mathrm{L}$ ). Electromyography revealed prominent myotonic discharges. The patient was suspected as having paramyotonia on the basis of clinical findings and electromyography examination. Subsequently, he was clinically diagnosed as having PMC. In order to confirm this 


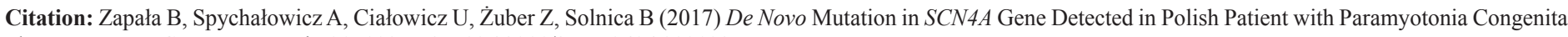
Phenotype. Int J Genom Data Min 01: 109. DOI: 10.29011/2577-0616.000109

clinical diagnosis, the patient and his parents were included in a molecular genetic study.

\section{Material and Methods}

After obtaining informed consent, we performed a mutational analysis of SCN $4 A$ gene in the patient and his parents. Whole blood samples for analysis were collected using tubes containing Ethylene Diamine Tetra Acetic Acid (EDTA). Total genomic DNA was extracted from peripheral blood leukocytes using High Pure PCR Template Preparation Kit (ROCHE Diagnostic, Mannheim, Germany). Sequences of primers specific to the $S C N 4 A$ gene presented according to Dian K. Nurputra et al. 2012 as previously described. The 24 SCN4A coding exons and adjacent intronic regions were amplified by polymerase chain reaction (PCR) with Fast Start PCR Master Kit (ROCHE Diagnostic). PCR was carried out using a PCR System Nexus Gsx 1e (Eppendorf). The sequences were determined on both DNA strands from at least two independent PCR products. The PCR products were purified with High Pure PCR Product Purification Kit (ROCHE Diagnostic) and sequenced by dideoxynucleotide chain termination using Big Dye Terminator v.3.1 Cycle Sequencing Kit (Thermofisher, Foster City, CA, USA). The products of this cycle sequencing were purified by unbound fluorescent dyes with Big Dye XTerminator Purification Kit (Thermofisher, Foster City, CA, USA) and separated in an automated DNA sequencer 3130xl Genetic Analyzer (Applied Biosystems, Foster City, CA, USA). The sequences were analysed by using AB DNA Sequencing Analysis Software v.5.2. (Applied Biosystems) and then were compared to the $S C N 4 A$ gene reference (NCBI Gene Bank Reference Sequence Accession Number: NM_000334.4 and ENSEMBL database Accession Number: ENST00000578147.5).

\section{Results}

Direct nucleotide sequence analysis of PCR products from the patient showed a $\mathrm{C}$ to $\mathrm{T}$ transition at nucleotide position 3938 in exon 22 c.[3938C $>$ T]; [3938C=] of the $S C N 4 A$ gene, resulting in the substitution of threonine for methionine at amino acid position 1313 in the SCN4A gene p.[Thrl313Met]; [Thr1313=] Figure 1. This heterogenous missense mutation as described above. All the nucleotides in that gene were examined, but no other mutations were detected (data not shown). The results of our analysis in the patient's mother and father showed no mutation in exon 22 of SCN4A Figure 1. Finally, based on these DNA results together with the observation of clinical symptoms the patient was diagnosed with PMC caused by a de novo mutation.

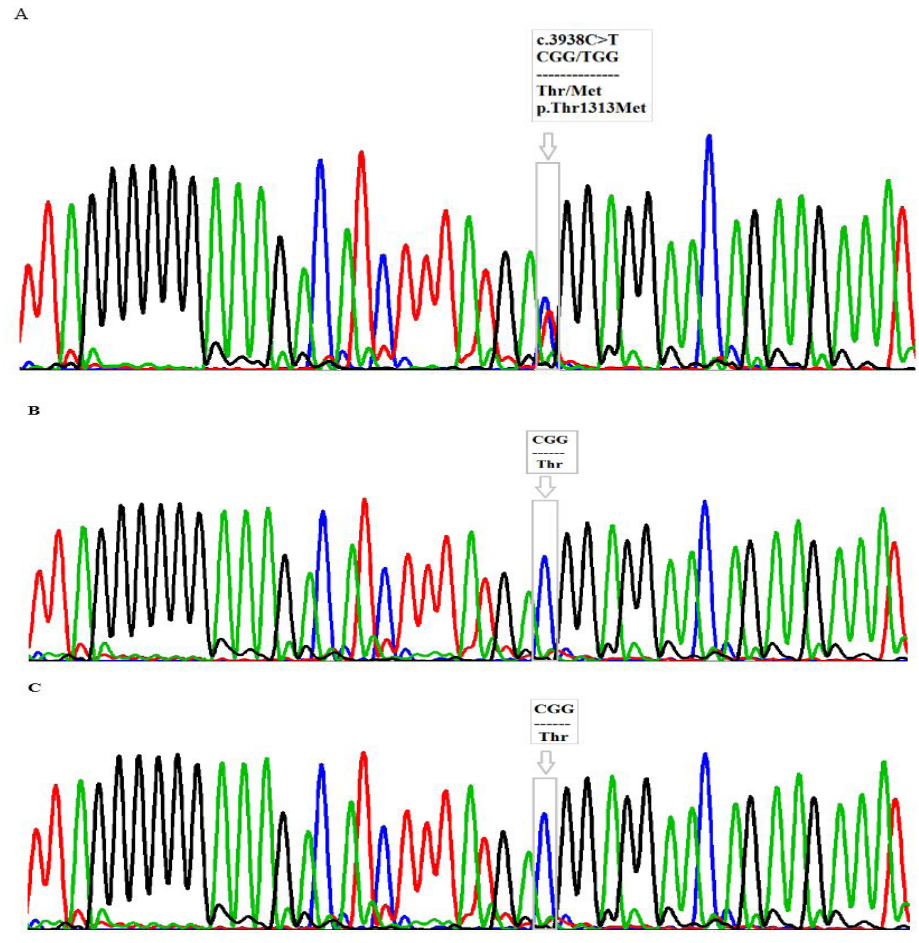

Figure 1: SCN $4 A$ sequencing chromatograms showing position 3939 of the coding DNA sequence (indicated by the grey frame). Graph A shows a de novo mutation in exon 22 identified in the proband where a substitution of cytosine to thymidine at position 3938 changes the codon for threonine at position 1313 into metionine. Graph B shows the SCN4A gene sequence chromatogram showing the nucleotide $\mathrm{C}$ at codon 3938 in the proband's mother. Graph C shows the $S C N 4 A$ gene sequence chromatogram showing the nucleotide $\mathrm{C}$ at codon 3938 in the proband's father.

Our patient with PMC phenotype has been shown to have the heterozygous missense de novo mutation c.[3938C $>\mathrm{T}]$; [3938C=], p.[Thr1313Met]; [Thr1313=] in the $S C N 4 A$ gene. This finding confirmed the clinical diagnosis of PMC in the patient. The mutation was present only in the patient and not in other family members (mother and father). Although it is known that PMC is a predominantly inherited neuromuscular disorder, our patient seems to be a sporadic case with no positive family history. It suggests that the disease in the patient was caused by a mutation that might have occurred in germ-line cells in one of the parents. Two mutations predominante in PMC in European populations: Thr1313Met (45\% of cases) [11] and Arg1448Cys (35\% of cases) 


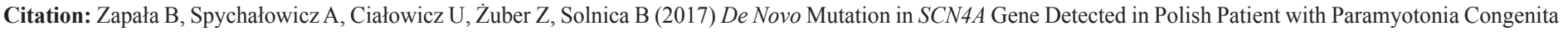
Phenotype. Int J Genom Data Min 01: 109. DOI: 10.29011/2577-0616.000109

[12]. Thr1313Met mutation was previously described and is known as being predominant in the population of France. In the Polish population, our diagnosis is the first such report of a mutation in SCN4A in a patient with PMC.

The SCN4A gene encodes the alpha subunit which plays a crucial role as a principal pore-forming and voltage-sensing subunit in the skeletal muscle sodium channels. Ion channels are transmembrane proteins that allow ions to flow into and out of the cells, creating electrical currents. The alpha-subunit comprises four repeated domains (from DI to DIV) as showed on Figure 2. Each domain consists of six transmembrane segments termed S1-S6. The fourth transmembrane segment (S4) of each domain contains positively charged amino acid and functions as voltage sensor for channel activation. In each domain, there is also an extracellular P-loop between the S5 and S6 segments. These P-loops together create ion-conducting pore, which acts as a selectivity filter. These filters are very important for ion permeability. The most crucial region of the alpha-subunit of the muscle sodium channel is an intracellular loop located between DIII and DIV domains. This is responsible for the rapid inactivation of this channel [13]. The alpha-subunit is also associated with the beta-subunit which has not been linked to any muscle human phenotype.

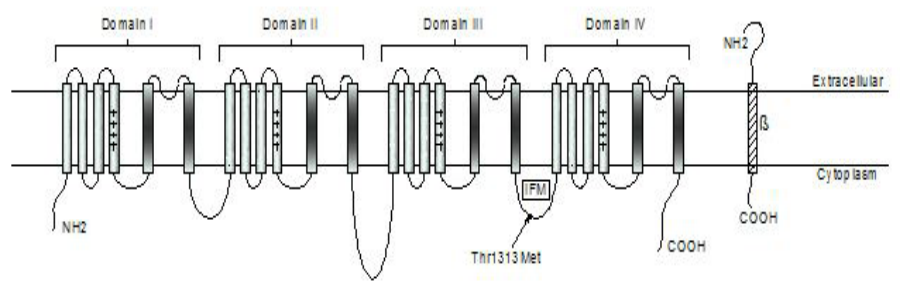

Figure 2: Two-dimensional structure of the rat Nav1.4 channel. A sodium channel is composed of four domains (I - IV), each consisting of six transmembrane segments. The -helical S4 segment, which possesses many positive charges, is the voltage sensor. The loop between domains III and IV is the inner mouth of the channel.

The mutations in the $S C N 4 A$ gene alter different features of the alpha-subunit of Nav 1.4. It has been shown that the principal defect of gating (opening and closing) is disruption to fast inactivation, rendering it delayed or incomplete. This results in channel re-openings and intracellular $\mathrm{Na}^{+}$accumulation, which depolarises muscle cells and generates repetitive action potentials. A mild depolarisation causes a long-lasting hyperexcitability, which leads to myotonia $[9,10,12]$.

In the PMC phenotype disruption to fast activation is the predominant abnormality of sodium channel gating. This fast inactivation is responsible for limitation of the action potential duration which initiates repolarization of the muscle fibers. The Thr1313Met mutation is situated in part of the protein relevant for channel inactivation in the intracellular loop between DIII and DIV domains. It thus causes the defective inactivation of the sodium channel. This results in myotonia and muscle stiffness. Moreover, in our patient both muscle stiffness and weakness were exacerbated by cold weather. It has been reported previously that Thr1313Met mutation results in temperature-sensitive behaviour of the sodium channel. In cultured human muscle cells was demonstrated that muscle fibers from PMC patients have normal membrane properties at $32^{\circ} \mathrm{C}$ while at $27^{\circ} \mathrm{C}$, they are inexcitable, exhibit increased conductivity of $\mathrm{Na}$, and have a reduced resting membrane potential of $-40 \mathrm{mV}$ [14].

In conclusion, in the Polish population, sodium channelopathy such as PMC is relatively rare. By mutational analysis of SCN4A gene, we identified a $\mathrm{C}$ to $\mathrm{T}$ transition at nucleotide position 3938 in exon 22 p.[Thr1313Met]; [Thr1313=] and we confirmed a diagnosis of PMC in a 16-year-old male suffering from coldinduced myotonia and stiffness. This is the first recorded diagnosis in the Polish population.

\section{Acknowledgements}

This research was funded by the Leading National Research Center, KNOW

\section{Conflict of Interest Disclosures}

The authors report no financial or other conflict of interest relevant to the subject of this article.

\section{Ethic Statement}

All procedures performed in studies involving human participants were in accordance with the ethical standards of the institutional and/or national research committee and with the 1964 Helsinki declaration and its later amendments or comparable ethical standards. Informed consent was obtained from all individual participants included in the study.

\section{References}

1. Eulenburg A (1886) Uber eine familiare, durch 6 Generationen ver folgbare Form congenitaler Paramyotonie. Neurol Centralbl 5: 265-272.

2. Bendahhou S, Cummins TR, Kwiecinski H, Waxman SG, Ptácek LJ (1999) Characterization of a new sodium channel mutation at arginine 1448 associated with moderate Paramyotonia congenita in humans. $J$ Physiol 518: 337-344.

3. Bendahhou S, Cummins TR, Tawil R, Waxman SG, Ptácek LJ (1999) Activation and inactivation of the voltage-gated sodium channel: role of segment S5 revealed by a novel hyperkalaemic periodic paralysis mutation. J Neurosci 19: 4762-4771.

4. Plassart E, Eymard B, Maurs L, Hauw JJ, Lyon-Caen O, et al. (1996) Paramyotonia congenita: genotype to phenotype correlations in two families and report of a new mutation in the sodium channel gene. $J$ Neurol Sci 142: 126-133. 
Citation: Zapała B, Spychałowicz A, Ciałowicz U, Żuber Z, Solnica B (2017) De Novo Mutation in SCN4A Gene Detected in Polish Patient with Paramyotonia Congenita Phenotype. Int J Genom Data Min 01: 109. DOI: 10.29011/2577-0616.000109

5. Koch MC, Baumbach K, George AL, Ricker K (1995) Paramyotonia congenita without paralysis on exposure to cold: a novel mutation in the SCN4A gene (Val1293lle). Neuroreport 6: 2001-2004.

6. Wu FF, Takahashi MP, Pegoraro E, Angelini C, Colleselli P, et al. (2001) A new mutation in a family with cold-aggravated myotonia disrupts $\mathrm{Na}(+)$ channel inactivation. Neurology 56: 878-884.

7. Hoffman EP, Lehmann-Horn F, Riidel R (1995) Overexcited or inactive: ion channels in muscle disease. Cell 80: 681-686.

8. Ashcroft FM (2000) Voltage-gated $\mathrm{Na}+$ channel, in: Ion Channels and Disease. Ashcroft FM, Ed. Academic Press, San Diego CA: 67-89.

9. Cannon SC (2001) Voltage-gated ion channelopathies of the nervous system. Clin Neurosci Res 57: 772-779.

10. Lehmann-Horn F, Jurkat-Rott K (1999) Voltage-gated ion channels and hereditary disease. Physiol Rev 79: 1317-1372.
11. McClatchey Al, Van den Bergh P, Pericak-Vance MA (1992) Temperature-sensitive mutations in the III-IV cytoplasmic loop region of the skeletal muscle sodium channel gene in paramyotonia congenita. Cell 68: 769-774

12. Plassart E, Eymard B, Maurs L, Hauw JJ, Lyon-Caen O, et al. (1996) Paramyotonia congenita: genotype to phenotype correlations in two families and report of a new mutation in the sodium channel gene. $J$ Neurol Sci 142: 126-133.

13. Armstrong CM (1997) A closer picture of the $\mathrm{K}$ channel gate from ion trapping experiments. J Gen Physiol 109: 523-524.

14. Boulos PT, Heiman-Patterson TD, Alexander GM, Tahmoush AJ (2000) Patch clamp studies of the thr1313met mutant sodium channel causing paramyotonia congenita. Muscle Nerve 23: 1736-1747. 